\title{
Simplified limbal approach in squint surgery
}

\author{
PETER FELLS \\ Department of Clinical Ophthalmology, Institute of Ophthalmology, and Moorfields Eye Hospital, ® \\ London
}

The limbal approach is not new to squint surgery* but, despite its considerable advantages in exposure and subsequent healing, the methods described so far are not easy to perform 0 to obtain the best final results. In particular, the incision commonly described needs very accurate suturing of the conjunctival flap with its two radially placed incisions to achieve a good cosmetic result. The method described below uses a triangular flap of $\gg$ conjunctiva which needs only a single limbal suture to restore limbal anatomy and is therefore much more easily performed.

\section{Operative technique}

The limbal fixation sutures (black nylon or Jameson Evans) are placed into episcleral tissue at 6 and 120 'clock and the eye is pulled across laterally to allow access to the medial rectus. The insertion of the medial rectus may be readily identified beneath the conjunctiva and a horizontal conjunctival incision is made just above and parallel to the upper border of medial rectus, some 3 or $4 \mathrm{~mm}$. from the limbus (Fig. I, I). Tenon's fascia is incised in the same plane and the wound is enlarged right up to the limbus in the I o'clock position. Tenon's fascia and conjunctiva are next cut away as one layer from the limbus, using non-pointed scissors, as far round as 5 o'clock. Lifting the combined Tenon's fascia and conjunctiva together, this triangular flap is dissected back, exposing the entire medial rectus insertion without any bleeding. It is important to take the limbal disinsertion of the conjunctiva as far round as 50 'clock, otherwise excessive traction on the conjunctival flap for exposure of the muscle may tear the conjunctiva away from the limbus in a radial direction. The muscle insertion may then be moved or dealt with in whatever way is necessary (Fig. I, 2).

When closing the conjunctiva, the apex of the triangular flap is identified and the flap held across its original site. It will almost invariably be found that if an apical suture were used then the conjunctiva would gape around the limbus. Holding the flap at the apex, it can readily be seen just where the suture must be placed along the originally limbal edge of the conjunctiva to ensure a snug fit around the limbus (Fig. I, 3). The second bite of this suture into the conjunctiva superiorly must first penetrate episcleral tissue and then emerge through the conjunctiva close to its edge. A large bite of conjunctiva which misses episcleral tissue tends to pull a hood of conjunctiva across the limbus. Normally a second suture will be needed in the horizontal extension of the incision, but occasionally this will be superfluous.

When a recession-resection operation is being performed, it is often convenient to expose both horizontal muscles before finally deciding how much to adjust the position of the muscle insertions. This applies particularly to second operations on the same eye. Using the fixation sutures at 12 and 6 o'clock as shown facilitates this. In any case it is often convenient to leave the suturing of both conjunctival flaps until the end of the operation. The 12 o'clock fixation suture is used to pull the 
eye slightly downwards, giving full access to the superior limbus from either side. Fig. I, 4 shows that, at the end of the operation, all four conjunctival sutures are beneath the upper lid and that no conjunctival scars are visible in the interpalpebral fissure. The limbal incision when healed is virtually undetectable.
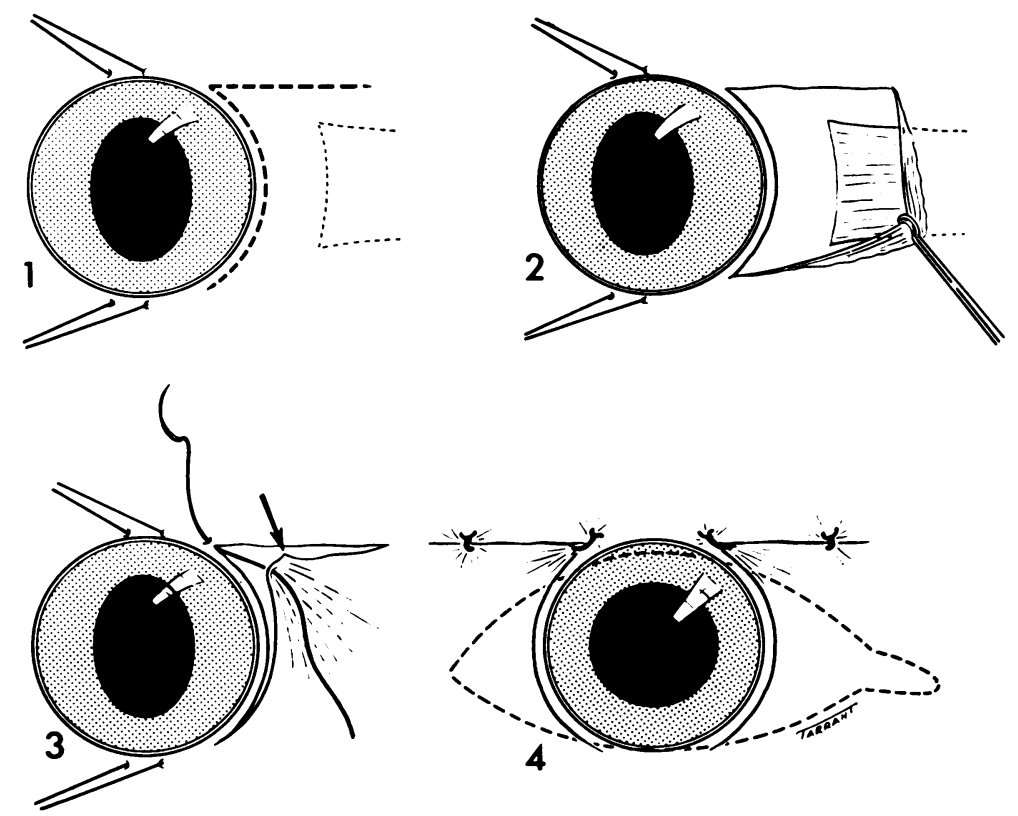

FIG. I Steps in a right medial rectus recession operation

(I) The faintly dotted line indicates the medial rectus beneath the conjunctiva. The heavily dashed line indicates the position and extent ( 1 to 5 o'clock) of the incision through conjunctiva and Tenon's fascia

(2) The flap of conjunctiva and Tenon's fascia allows full exposure of the medial rectus insertion. Note that extending the limbal incision far enough inferiorly prevents the conjunctiva from tearing away radially

(3) Suturing the conjunctival flap back. Note that the suture does not pass through the original apex of the flap (arrowed) but lower down the originally limbal edge to give an accurate limbal fit to the conjunctiva

(4) Final appearance after a recession-resection operation with four conjunctival sutures all lying beneath the normally opened upper lid. The dashed line indicates the interpalpebral aperture

Throughout a normal squint operation a 6-o plain collagen suture is used. This allows for reattaching both muscles and for the conjunctival suturing. Because this suture is soluble and the conjunctival sutures are placed beneath the upper lid, the patient suffers the minimum of discomfort, and this method has proved very satisfactory in children and young adults.

This limbal approach is not used for vertical rectus muscle surgery, nor for oblique muscle operations as there is no virtue in bringing a well-hidden conjunctival wound into the interpalpebral opening.

The appearance of an operated eye 24 hours after a medial rectus recession is shown in Figs 2 and 3 (overleaf).

\section{Summary}

A method of approaching extraocular muscle surgery in cases of squint by way of the limbus is described and illustrated. 


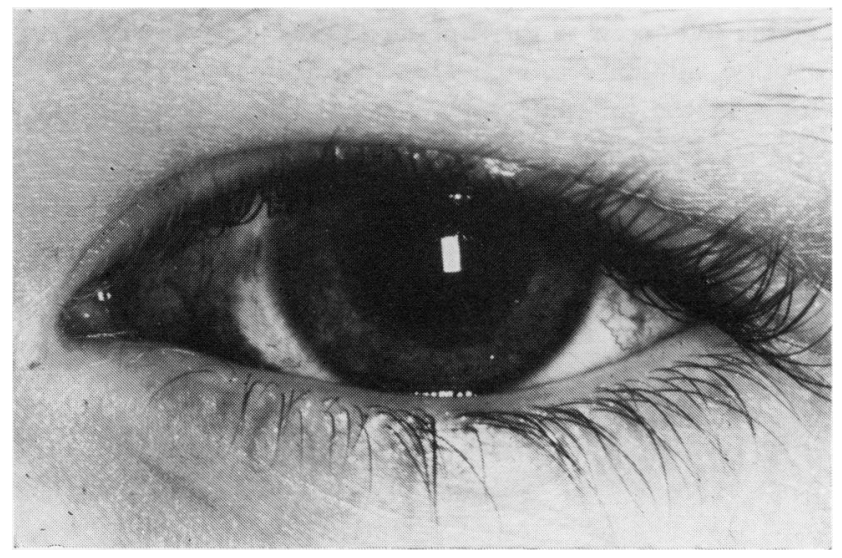

Peter Fells
FIG. 2 Left eye 24 hours after a

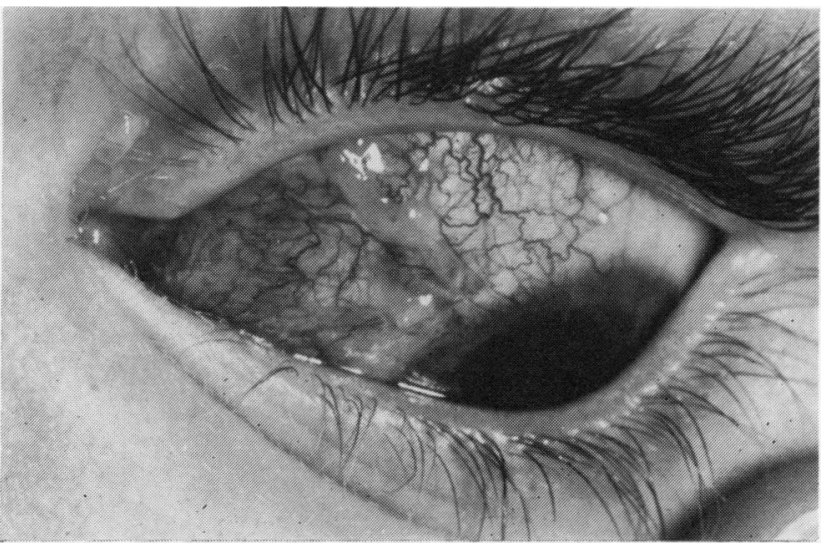

medial rectus recession in a 5-yearold boy

FIG. 3 Same eye as in Fig. 2 looking down to show the sutured conjunctiva. There are two sutures with some mucus around them

\section{References}

CORTes v., м. (1962) Arch. chil. Oftal., 19, 54

massin, M., and hUdelo, J. (I962) Ann. Oculist. (Paris), 195, 995 MORENO DE MULET, E. I. (1964) 7. pediat. Ophthal., r, July, p. 25 NOORDEN, G. K. von (1968) Arch. Ophthal. (Chicago), 80, 94 (1969) Ibid., 82, 349 